\title{
Cholangiocarcinoma: Challenges and Improving the Outcomes
}

\author{
Suhang Verma ${ }^{1} \quad$ Jayanta Samanta ${ }^{1} \quad$ Anupam K. Singh ${ }^{1} \quad$ Rakesh Kochhar $^{1}$ \\ ${ }^{1}$ Department of Gastroenterology, Postgraduate Institute of \\ Medical Education and Research, Chandigarh, India
}

J Digest Endosc 2020;10:210-212

Cholangiocarcinoma (CCA) remains the second most common primary biliary malignancy globally and in India after adenocarcinoma of the gallbladder, with a rising incidence. ${ }^{1}$ It is an aggressive neoplasia arising from the biliary epithelium, resulting in biliary strictures/mass and progressive obstructive jaundice. It may be further classified based on the location; perihilar (50\%) remains the most common site followed by distal (40\%) and intrahepatic (10\%). ${ }^{2}$

Global incidence ranges from 0.3 to 85 per 100,000 population, with highest rates in Southeast Asia. ${ }^{3}$ The mean age of presentation is in the seventh decade of life, with a slight male predominance. However, a younger onset may be seen in conditions like primary sclerosing cholangitis and Caroli's disease. ${ }^{4}$ Important risk factors include environmental exposures (smoking, alcohol, asbestos, and thorotrast), parasites (Clonorchis and Opisthorchis), chronic liver disease (cirrhosis, chronic viral hepatitis, hemochromatosis, and primary sclerosing cholangitis), hepatolithiasis, and choledochal cysts. However, no etiology can be found despite all efforts in 3 half of the cases. ${ }^{3}$

The most common presentation is development of a progressive (predominantly painless) jaundice (90\%), followed by abdominal pain and weight loss (35\%) while acute cholangitis is uncommon (10\%). Eventually, patients progress to liver failure or die due to metastases. However, symptoms may be nonspecific and depend on the site of the tumor. ${ }^{5}$ Diagnosis is challenging and often requires a high degree of clinical suspicion in an appropriate setting and a multipronged, multimodality approach (clinical, biochemical, radiological, and endoscopic) is needed to reach an accurate diagnosis.

Despite all efforts, survival in CCA has not seen much improvement over last several decades. Despite being a slow-growing, paucicellular malignancy, it remains clinically silent till an advanced stage. Nearly $50 \%$ of the patients are stage III while 10 to $20 \%$ are stage IV at presentation. ${ }^{6}$ The prognosis is usually guarded and the therapeutic armamentarium is limited to palliative measures in most of the patients. Complete surgical resection remains the only curative therapy in CCA; however, only 20 to $30 \%$ patients are candidates for resection. ${ }^{7}$ Many patients are also rendered unfit for surgery because of the poor performance status and associated comorbidities.

Even in patients who undergo curative resection, 5-year survival rates are low (9-18\% for proximal lesions and $20-30 \%$ for distal lesions). ${ }^{6}$ Achieving R0 resection is difficult and recurrence rates are high. However, some centers have achieved 5-year survival rates as high as 35\% in patients with perihilar CCA with an aggressive resection strategy. ${ }^{8}$ Surgical morbidity and mortality also remains high. Orthotropic liver transplantation following neoadjuvant chemoradiation has shown promise in selected patients with unresectable perihilar CCA, with 5-year disease-free survival of up to $65 \% .^{9}$ The role of adjuvant chemoradiotherapy after curative resection is uncertain at present, with a recent trial using gemcitabine and oxaliplatin failing to demonstrate a benefit. ${ }^{10}$

Unresectable patients are candidates for palliative therapy and do worse, with high mortality rates. In a large retrospective study, the median survival in patients with unresectable CCA was a mere 3.9 months. ${ }^{11}$ Palliative therapy comprises systemic chemotherapy, local chemoradiotherapy, and biliary drainage, apart from other supportive care. The primary aim is to alleviate troublesome symptoms and improve the quality of life rather than focusing only on survival. ${ }^{6}$ CCA is a relatively radiochemoresistant tumor by nature with poor response. Using single- and multiagent chemotherapy (most of them based on 5-fluorouracil), partial response rates of up to 18 to $38 \%$ have been obtained with reported median survival of 2 to 15 months. ${ }^{5}$ Currently a combination therapy utilizing gemcitabine and cisplatin is considered as the first-line chemotherapy in patients with unresectable CCA. In a phase 3 randomized trial comparing a combination of gemcitabine and cisplatin with gemcitabine alone in patients with locally advanced or metastatic biliary tract cancers, the median overall survival (11.7 months vs. 8.1 months, $p$-value $<0.001$ ) and

\footnotetext{
Address for correspondence

Dr. Rakesh Kochhar, MD, DM, Department of Gastroenterology, Postgraduate Institute of Medical Education and Research, Chandigarh 160012, India (e-mail: dr_kochhar@hotmail.com).
}

DOI https://doi.org/ 10.1055/s-0040-1709910 ISSN 0976-5042.
(C)2020 Society of Gastrointestinal Endoscopy of India

\section{License terms}

(ㅇ)(1) $\Theta \circledast$ 
median progression-free survival ( 8 months vs. 5 months, $p$-value $<0.001$ ) were significantly better in the combination group without any excess $3 .^{12}$ Performance status of patients is the most important consideration before commencing palliative chemotherapy as moribund patients are unlikely to tolerate or show benefit. For the patients who are unable to tolerate combination chemotherapy, gemcitabine alone or a 5 -fluorouracil-based therapy may be administered..$^{13}$

Other forms of local therapy that have been evaluated and found to be of benefit in locally advanced unresectable CCA include transarterial chemotherapy (TACE), transarterial radiotherapy (TARE), and external-beam radiotherapy (EBRT), and may be used in patients unfit for systemic therapy. Median survival rates of up to 12 to 15 months with TACE and 11 to 22 months with TARE have been obtained in locally advanced CCA. In some cases, downstaging may be achieved facilitating curative resection. ${ }^{1}$ Due to a lack of satisfactory response with the currently available therapeutic options and better understanding of the pathophysiological and genetic alterations operating in CCA, a large number of targeted and molecular therapy (including immunotherapy) are being evaluated in trials. ${ }^{1}$

A palliative biliary drainage can reduce pain, pruritus, risk of liver failure, cholangitis, improve quality of life, and facilitate administration of systemic chemotherapy. ${ }^{14}$ The choice of route (percutaneous vs. endoscopic) is based on site of obstruction, availability of expertise, and patient preference. Metallic stents have superiority over plastic stents with higher success rates, lower complications, and reinterventions. Overall cost effectiveness is also better if a survival for greater than 4 to 6 months is anticipated. ${ }^{15}$ Drainage of more than $50 \%$ liver volume (by using multiple/bilateral stents) may be superior with better reduction of bilirubin, lower risk of cholangitis, and improved survival. ${ }^{16}$ Bismuth type I and II CCA may be better tackled via endoscopic route, whereas a percutaneous route may be better suited for Bismuth type III and IV lesions. ${ }^{7}$ Radiofrequency ablation (RFA) and photodynamic therapy (PDT) are upcoming adjuvants to endotherapy, that not only improve the stent patency rates but can also improve the survival in patients with CCA. ${ }^{17,18}$

In this issue of Journal of Digestive Endoscopy, Sudhakar et $\mathrm{al}^{19}$ have described the clinical profile and outcomes of patients with unresectable cholangiocarcinoma (CCA) in Southern India. It was a retrospective, single-center study conducted between 2013 and 2016 and included 86 patients. Most of the patients were diagnosed at an advanced stage; only $16 / 86$ (18.6\%) underwent curative resection while the remaining were deemed unresectable and underwent palliative therapy only. Despite having limitations of being a single-center, retrospective study with a small sample size, the study is valuable for being the first study that has described the natural history of unresectable CCA in Indian patients.

The cohort in current study was younger (mean age 53.43 years) as compared with global average. The reasons why younger a younger population in India is developing CCA remains unclear. Tobacco, alcohol, cirrhosis, and hepatitis $C$ virus were the most important risk factors. The most common presentation was obstructive jaundice (85.7\%), pain was infrequent (21.4\%), and cholangitis (8.5\%) was uncommon. Mean serum bilirubin level was $9.7 \mathrm{mg} / \mathrm{dL}$ while serum alkaline phosphatase (ALP) was not very high (298 U/L). Hilar CCA (58.57\%) was most common type followed by distal CCA (30\%) and intrahepatic CCA (11.4\%). Most common route of palliative biliary drainage was endoscopic (74.28\%), followed by percutaneous ( $25.71 \%$ ) while none were offered chemoradiotherapy. Overall prognosis was poor with a mean survival of 6 months. The patients with higher bilirubin level performed significantly worse.

However, certain issues need to be addressed. One of the most glaring findings is that none of the patients were offered any palliative chemotherapy despite undergoing palliative biliary drainage. The reasons have not been explained and it remains a major drawback of the study. As mentioned before, palliative systemic chemotherapy has been accepted as the standard of care in management of unresectable cholangiocarcinoma, although poor performance status and high bilirubin levels may have hindered its administration. At least a subgroup of patients with unresectable CCA may have been candidates for liver transplantation.

Biliary drainage remains one of the cornerstones of palliative therapy of CCA and was performed in all (100\%) the patients in current study. The authors have not mentioned the impact of location and type of stricture in deciding the route (percutaneous vs. endoscopic), strategy (unilateral vs. bilateral), or modality (plastic vs. metallic stent) of biliary drainage. Although the study found no significant difference in survival of patients with endoscopic or percutaneous drainage, the study also provided an opportunity to provide the details pertaining to the technical and clinical success, failure rates, complications, and need for reintervention. It is also important to realize the futility of drainage in at least a subgroup of patients with more advanced disease and multilevel block who are unlikely to be benefited and are likely to have higher complication rates. The selection of candidates and planning the optimal drainage strategy is imperative to obtain better outcomes.

To conclude, CCA remains a malignancy with poor outcomes and most patients at presentation are candidates of palliative therapy only. There is a lack of data from India; however, our patients are much younger than those in the west. Survival in advanced cases is 3 to 6 months. Biliary drainage should be offered when indicated to improve quality of life and facilitate chemotherapy. Survival may be improved by the systemic and local therapy options currently available and enrolment of patients in trials should be encouraged.

\section{Conflict of Interest}

None declared.

\section{References}

1 Rizvi S, Khan SA, Hallemeier CL, Kelley RK, Gores GJ. Cholangiocarcinoma - evolving concepts and therapeutic strategies. Nat Rev Clin Oncol 2018;15(2):95-111

2 DeOliveira ML, Cunningham SC, Cameron JL, et al. Cholangiocarcinoma: thirty-one-year experience with 564 patients at a single institution. Ann Surg 2007;245(5):755-762 
3 Khan SA, Tavolari S, Brandi G. Cholangiocarcinoma: epidemiology and risk factors. Liver Int 2019;39(Suppl 1):19-31

4 Razumilava N, Gores GJ. Cholangiocarcinoma. Lancet 2014;383(9935):2168-2179

5 Olnes MJ, Erlich R. A review and update on cholangiocarcinoma. Oncology 2004;66(3):167-179

6 Khan SA, Davidson BR, Goldin R, et al; British Society of Gastroenterology. Guidelines for the diagnosis and treatment of cholangiocarcinoma: consensus document. Gut 2002;51(Suppl 6):VI1-VI9

7 Goenka MK, Goenka U. Palliation: hilar cholangiocarcinoma. World J Hepatol 2014;6(8):559-569

8 Kosuge T, Yamamoto J, Shimada K, Yamasaki S, Makuuchi M. Improved surgical results for hilar cholangiocarcinoma with procedures including major hepatic resection. Ann Surg 1999;230(5):663-671

9 Darwish Murad S, Kim WR, Harnois DM, et al. Efficacy of neoadjuvant chemoradiation, followed by liver transplantation, for perihilar cholangiocarcinoma at 12 US centers. Gastroenterology 2012;143(1):88-98

10 Edeline J, Benabdelghani M, Bertaut A, et al. Gemcitabine and oxaliplatin chemotherapy or surveillance in resected biliary tract cancer (PRODIGE 12-ACCORD 18-UNICANCER GI): a randomized phase III study. J Clin Oncol 2019;37(8):658-667

11 Park J, Kim MH, Kim KP, et al. Natural history and prognostic factors of advanced cholangiocarcinoma without surgery, chemotherapy, or radiotherapy: a large-scale observational study. Gut Liver 2009;3(4):298-305
12 Valle J, Wasan H, Palmer DH, et al; ABC-02 Trial Investigators. Cisplatin plus gemcitabine versus gemcitabine for biliary tract cancer. N Engl J Med 2010;362(14):1273-1281

13 Patel T. Cholangiocarcinoma-controversies and challenges. Nat Rev Gastroenterol Hepatol 2011;8(4):189-200

14 Madhusudhan KS, Gamanagatti S, Gupta AK. Imaging and interventions in hilar cholangiocarcinoma: a review. World J Radiol 2015;7(2):28-44

15 Sangchan A, Kongkasame W, Pugkhem A, Jenwitheesuk K, Mairiang P. Efficacy of metal and plastic stents in unresectable complex hilar cholangiocarcinoma: a randomized controlled trial. Gastrointest Endosc 2012;76(1):93-99

16 Vienne A, Hobeika E, Gouya H, et al. Prediction of drainage effectiveness during endoscopic stenting of malignant hilar strictures: the role of liver volume assessment. Gastrointest Endosc 2010;72(4):728-735

17 Kahaleh M, Mishra R, Shami VM, et al. Unresectable cholangiocarcinoma: comparison of survival in biliary stenting alone versus stenting with photodynamic therapy. Clin Gastroenterol Hepatol 2008;6(3):290-297

18 Yang J, Wang J, Zhou H, et al. Efficacy and safety of endoscopic radiofrequency ablation for unresectable extrahepatic cholangiocarcinoma: a randomized trial. Endoscopy 2018;50(8):751-760

19 Sudhakar A, Sravan Kumar K, Girinadh LRS, MuraliKrishna P, Natural history of patients with unresectable cholangiocarcinoma. J Digest Endosc 2019;10:206-209 